\title{
Behavior of 3,4-Endiol Form of 2,3-Diketo-Gulono- $\delta$-Lactone Formed from Dehydro-L-Ascorbic Acid in Deoxygenated and Neutral Solution
}

\author{
Masanosuke TAKAgI, Atsushi KaWAJIRI, Kunihiko NAKATA, \\ and Naofumi MORITA ${ }^{1}$ \\ Laboratory of Food Chemistry, College of Agriculture, \\ University of Osaka Prefecture, \\ Sakai, Osaka 591, Japan
}

(Received September 7, 1988)

\begin{abstract}
Summary The formation of L-ascorbic acid (AsA) was observed when dehydro-L-ascorbic acid (DHA) was dissolved in neutral buffer solutions under $\mathrm{N}_{2}$ bubbling at room temperature. The reduction of DHA was done with the lactonized compound of 2,3-diketo-L-gulonic acid (DKG), that is, the 3,4-endiol form of 2,3-diketo-gulono- $\delta$-lactone (3,4-End DKGL). 3,4End DKGL was formed from DHA or DKG (yield about $10 \%$ ) under $\mathrm{N}_{2}$ bubbling in neutral buffer solution ( $\mathrm{pH}$ 7.2). This material was not stable in neutral or alkaline solutions. 3,4-End DKGL suppressed more strongly the linoleic acid (LA) peroxidation in the medium containing $20 \% \mathrm{EtOH}$ and $10 \mathrm{~mm}$ LA than did AsA. This may suggest the possibility that 3,4-End DKGL reproduces AsA from DHA in physiological status.

Key Words dehydro-L-ascorbic acid, 2,3-diketo-L-gulonic acid, 3,4-endiol form of 2,3-diketo-gulono- $\delta$-lactone, reduction of DHA, isomerization of oxidized AsA, suppression of linoleic acid peroxidation
\end{abstract}

L-Ascorbic acid (AsA) is not very stable in neutral or alkaline solutions. AsA is easily oxidized by active oxygen species, UV light, etc., and the oxidized form of AsA, that is, dehydro-L-ascorbic acid (DHA), is reported to accelerate lipid peroxidation $(1,2)$. The lactone ring of DHA is split rather easily in neutral to alkaline solutions, followed by formations of 2,3-diketo-L-gulonic acid (DKG) and its further degraded or transformed products, as reported by Otsuka et al. $(3,4)$. The products are easily decomposed and the decomposition reactions are generally irreversible, but splitting products of DHA or $\operatorname{DKG}(5,6)$ did not accelerate the peroxidation of linoleic acid (LA), as reported (7).

When an aqueous solution of DHA was left overnight at room temperature under $\mathrm{N}_{2}$ bubbling, two substances having AsA-like reducing activity (designated $h_{1}$ and $h_{2}$ ) were obtained. The $h_{2}$ had a more negative oxidation potential on the

1 高木正之助, 川尻厚志，中田邦彦，森田尚文 
polarogram than did AsA $(8,9)$. This indirectly suggests that a more easily oxidizable substance was formed from DHA under the present experimental conditions, and also that some kind of derivative having a stronger reducing activity than that of AsA, which was presumably derived from DHA, would reduce DHA to AsA in the reaction mixture. From this point of view, we wanted to study the degradation mechanism or pathway of DHA or DKG in alkaline or neutral solutions.

The fact that DHA was reduced in neutral solution under $\mathrm{N}_{2}$ bubbling is interesting with regard to the action of vitamin $\mathrm{C}$ under physiological conditions $(10,11)$. In this study, we describe the characterization of this electrooxidizable substance.

\section{EXPERIMENTAL}

Chemicals. Linoleic acid (LA) was purchased from Sigma Chemical Co., and diluted to $100 \mathrm{~mm}$ with freshly distilled $\mathrm{EtOH}$ under $\mathrm{N}_{2}$ bubbling, and stored at $-20^{\circ} \mathrm{C}$ until use. DHA and DKG were prepared from AsA, as reported previously $(1,6)$. Dithiothreitol (DTT) was purchased from Wako Pure Chemical Industries, Ltd.

Analytical methods. All evaporations were carried out below $40^{\circ} \mathrm{C}$ under reduced pressure. High-performance liquid chromatography (HPLC) was done with an Irica chromatography apparatus (P-500) as the pump system, with a spectrophotometric detector (P-553), an amperometric detector (E-502), and a column $(4 \times 250 \mathrm{~mm})$ of Irica RP-18. The column was eluted with a solvent system of $\mathrm{CH}_{3} \mathrm{OH}-\mathrm{H}_{2} \mathrm{O}$-acetic acid $(30: 370: 4, \mathrm{v} / \mathrm{v})$ at a flow rate of $0.6 \mathrm{ml} / \mathrm{min}$. The sample was monitored photometrically (mainly at $245 \mathrm{~nm}$ ) and amperometrically at an applied potential of $0.5 \mathrm{~V}$ ( $v s$. an $\mathrm{Ag} / \mathrm{Ag}^{+}$electrode). Gas chromatography and mass spectrometry (GC-MS) was done with a Hitachi RMU-6MG apparatus. Polarography was done with an apparatus of Yanagimoto P-1000, as reported previously $(7,8)$. DHA $(\sim 100 \mathrm{~mm})$ in $0.5 \mathrm{M}$ phosphate buffer $(\mathrm{pH} 7.2)$ was reduced with a fivefold excess of DTT at $37^{\circ} \mathrm{C}$ for $10 \mathrm{~min}$. The reduced form of DHA, namely AsA, was analyzed by thin-layer chromatography (TLC) using a solvent mixture of $\mathrm{CH}_{3} \mathrm{CN}-\mathrm{H}_{2} \mathrm{O}$-acetone-acetic acid $(80: 15: 5: 1, \mathrm{v} / \mathrm{v})$, or by HPLC using an RP-18 column as described above. Nuclear magnetic resonance (NMR) was done with a JEOL apparatus GSX-270 in $\mathrm{CD}_{3} \mathrm{OD}$ using tetramethylsilane (TMS) as the internal standard. The IR spectrum was taken on a JASCO IRA-2 spectrometer, and the UV spectra were recorded on a JASCO Ubest-30 spectrophotometer.

Peroxidation of $L A$. Degraded products of AsA or DHA were tested for the peroxidation of LA, as reported previously $(1,2)$ : the reaction mixture contained $0.5 \mathrm{ml}$ of $100 \mathrm{~mm}$ LA in EtOH, $0.5 \mathrm{ml}$ of EtOH, $4 \mathrm{ml}$ of phosphate buffer $(0.1 \mathrm{M}, \mathrm{pH}$ 6.8 ), and $0.1 \mathrm{ml}$ of the material to be tested, as catalyst. The concentration of the catalyst was $135 \mu \mathrm{M}$ unless otherwise stated. A portion $(0.3 \mathrm{ml})$ of the sample was 
withdrawn at specified times, then $3 \mathrm{ml}$ of $60 \%$ EtOH aqueous solution containing $200 \mathrm{ppm}$ EDTA was added. After this solution had been vigorously shaken at $37^{\circ} \mathrm{C}$, the UV spectra were taken in the range of $200-300 \mathrm{~nm}$ : the absorbances at 265 and $233 \mathrm{~nm}$ were taken for the remaining AsA and LA hydroperoxide formed, respectively.

\section{RESULTS AND DISCUSSION}

\section{Reduction of DHA by DTT}

Experimental conditions for the reduction of DHA to AsA were tested. Excess amounts of DTT (up to 25-fold excess) dissolved in phosphate buffer ( $0.2 \mathrm{M}, \mathrm{pH} 7.2$ ) were added to $100 \mathrm{~mm}$ DHA solution ( $\mathrm{pH} 7.2$ ), and left for $1 \mathrm{~h}$ at $20^{\circ} \mathrm{C}$. A fivefold excess of DTT was sufficient for the reduction of DHA. The effects of $\mathrm{pH}$ on the reduction of DHA with DTT were tested at the same temperature. For the reduction of DHA, neutral ranges from 6.5 to 9 were the most favorable, and only a weak activity was observed below $\mathrm{pH} 4$.

For the test of stability of DHA in solution, $100 \mathrm{~mm}$ DHA in various buffer solutions was left for $1 \mathrm{~h}$ at $37^{\circ} \mathrm{C}$ under $\mathrm{N}_{2}$ bubbling. Thereafter, a portion of the solution was withdrawn, and the amount of AsA formed was measured by HPLC. Another portion was used to assay the remaining DHA by the addition of DTT $(500 \mathrm{~mm})$ in phosphate buffer, and left for $10 \mathrm{~min}$ at room temperature. The concentration of DHA remaining was calculated by the difference before and after DTT reduction of the testing solution. Table 1 shows the effects of $\mathrm{pH}$ on the degradation of DHA and the formation of AsA. DHA was relatively stable in acidic solution (around $\mathrm{pH} 4$ ), but AsA was formed in neutral to alkaline solutions.

Table 1. Effects of $\mathrm{pH}$ on the degradation of DHA and the formation of AsA.

\begin{tabular}{ccc}
\hline $\mathrm{pH}$ & $\begin{array}{c}\text { DHA remaining } \\
(\mathrm{mM})\end{array}$ & $\begin{array}{c}\text { AsA formed } \\
(\mathrm{mM})\end{array}$ \\
\hline 3.7 & 35.4 & - \\
4.7 & 32.0 & - \\
5.7 & 13.5 & 4.3 \\
6.7 & 2.1 & 11.0 \\
7.7 & - & 7.4 \\
9.2 & - & 3.3 \\
9.7 & - & 2.9 \\
\hline
\end{tabular}

DHA aqueous solution was added to various buffers to make the final concentration of $100 \mathrm{~mm}$ under bubbling of $\mathrm{N}_{2}$ gas. After shaking for $1 \mathrm{~h}$ at $37^{\circ} \mathrm{C}$, a portion of the reaction mixture was withdrawn, and the concentration of AsA was measured by HPLC; that of DHA remaining a was estimated from the response of AsA on the chromatogram of HPLC by subtraction of the values before and after DTT reduction of the testing solution. 


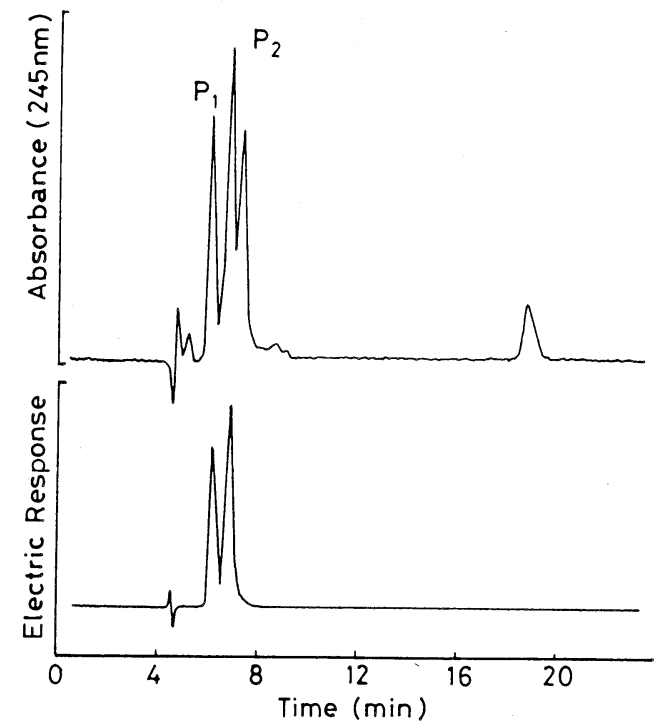

Fig. 1. HPLC of the degraded products of DHA in phosphate buffer. $100 \mathrm{~mm}$ DHA in phosphate buffer ( $\mathrm{pH} 7.2$ ) was left for $1 \mathrm{~h}$ at $37^{\circ} \mathrm{C}$ under $\mathrm{N}_{2}$ bubbling. A portion of the sample was analyzed by HPLC, monitoring photometrically at $245 \mathrm{~nm}$, and amperometrically at $500 \mathrm{mV}$ of applied potential. Other conditions are described in the text. $\mathrm{P}_{1}$, AsA; $\mathrm{P}_{2}, 3,4-$ End DKGL.

\section{Detection of oxidizable substance in neutral solution}

To detect degraded products during mild degradation, $100 \mathrm{~mm}$ DHA aqueous solution was left overnight at the ambient temperature under $\mathrm{N}_{2}$ bubbling. A portion of the sample was checked by TLC (data not shown). Four kinds of spots having reducing activity were detected by the spraying of ethanolic 2,6-dichlorophenol indophenol (DCIP) solution onto the plate. The major spot corresponding to an $R_{f}$ value of 0.5 was found to be AsA, but that of $R_{f} 0.6$ coincided with the $3,4-$ endiol form of 2,3-diketo-gulono- $\delta$-lactone (3,4-End DKGL), as reported by Otsuka et al. $(3,4)$. Another portion of the reaction mixture was analyzed by HPLC using $\mathrm{CH}_{3} \mathrm{OH}$-water-acetic acid $(30: 370: 4, \mathrm{v} / \mathrm{v})$. Since 3 ,4-End DKGL had the similar absorption peak to AsA, the absorption peaks at 265 and $245 \mathrm{~nm}$ were used for the estimation of both compounds in alkaline and acidic solutions, respectively (3). As is obvious from Fig. 1, two main peaks $\left(\mathrm{P}_{1}\right.$ and $\left.\mathrm{P}_{2}\right)$ were observed. Both $\mathrm{P}_{1}$ and $\mathrm{P}_{2}$ were positive by the detection of the electrochemical detector. $\mathrm{P}_{1}$ coincided with authentic AsA, whereas $\mathrm{P}_{2}$ was considered to be 3,4-End DKGL.

\section{Preparation of 3,4-End DKGL}

For the preparation of the substance indicated as $P_{2}$, HPLC may be most convenient from the viewpoint of purity of the sample. But this HPLC method is not practical for obtaining a sufficient amount of sample for various tests, if a big 

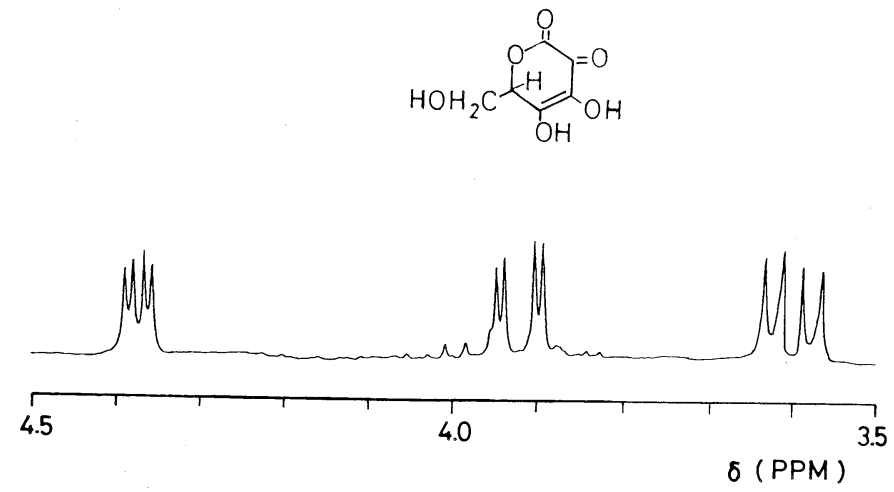

Fig. 2. ${ }^{1} \mathrm{H}-\mathrm{NMR}$ spectrum of 3,4-End $\mathrm{DKGL}$ in $\mathrm{CD}_{3} \mathrm{OD}$ at $270 \mathrm{MHz}$.

column is not available for the preparation. In this experiment, we used preparative precoated-TLC plates under a nitrogen stream at room temperature. The main products corresponding to $R_{f} 0.6$ were extracted from the wetted plates using the same solvent mixture as for development. The extract was evaporated to a syrup under reduced pressure below $30^{\circ} \mathrm{C}$, and the syrup was used for the following analyses. To a portion of the syrup was added solid $\mathrm{KBr}(100 \mathrm{mg})$, and an IR spectrum of the sample was taken after complete evaporation to remove remaining acetic acid in the syrup. The IR spectrum was the same as that of 3,4-End DKGL, as reported by Otsuka et al. $(3,4)$.

Figure 2 shows the ${ }^{1} \mathrm{H}$ NMR spectrum of the syrup in $\mathrm{CD}_{3} \mathrm{OD}$. In the ${ }^{1} \mathrm{H}$ NMR spectrum, the signals of double doublet centered at $3.60 \mathrm{ppm}\left(1 \mathrm{H}, J_{5,6 \mathrm{~b}}=\right.$ $6.20 \mathrm{~Hz})$ and $3.92 \mathrm{ppm}\left(1 \mathrm{H}, J_{6 \mathrm{a}, 6 \mathrm{~b}}=12.1 \mathrm{~Hz}\right)$ were assigned to the side chain residue at the ring structure of 3,4-End DKGL. On the other hand, the signals of double doublet centered at $4.38 \mathrm{ppm}\left(1 \mathrm{H}, J_{5,6 \mathrm{a}}=2.75 \mathrm{~Hz}\right)$ were assigned to $\mathrm{H}-5$ of the sixmembered lactone ring. As a result, this compound was considered to be 3,4-End DKGL, combining the agreement of the $R_{f}$ values, the fragmentation patterns by GC-MS, and some other analytical data, as has been reported previously (3).

\section{Formation of 3,4-End DKGL from DHA or DKG}

DHA or DKG was dissolved in $0.1 \mathrm{M}$ phosphate buffer ( $\mathrm{pH} 7.2$ ) and tested for the formation of 3,4-End DKGL under $\mathrm{N}_{2}$ bubbling at $37^{\circ} \mathrm{C}$ with gentle shaking. A portion of the sample was withdrawn at the specified times, and analyzed by HPLC. Figure 3 shows the course of 3,4-End DKGL formation from 1 and $5 \mathrm{~mm}$ DKG. For the measurement of the 3,4-End DKGL formed from DKG, the peak area of 3,4-End DKGL relative to that of AsA was calculated from the chromatogram of HPLC. The concentration of 3,4-End DKGL was maximum after $30 \mathrm{~min}$ of incubation, and the yield was about $10 \%$ from 1 or $5 \mathrm{~mm} \mathrm{DKG} \mathrm{used.} \mathrm{Thereafter} \mathrm{the}$ amounts decreased slightly with time. In the case of DHA, almost the same results were observed as for DKG; a somewhat longer lag period was required for the case 


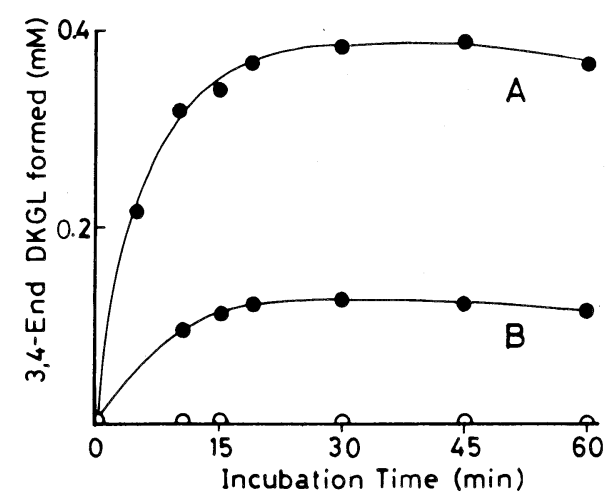

Fig. 3. Course of formation of 3,4-End DKGL from DKG. Solid DKG was dissolved in $0.5 \mathrm{M}$ phosphate buffer ( $\mathrm{pH} 7.2$ ) to make the final concentration of 1 and $5 \mathrm{~mm}$. After standing for the given times, a portion of the sample was analyzed by HPLC, monitoring photometrically at $245 \mathrm{~nm}$. Other conditions are described in the text. O, AsA formed; - 3,4-End DKGL formed from $5 \mathrm{~mm}$ DKG (A), 1 mм DKG (B).

of DHA, because the formation of 3,4-End DKGL proceeds via the hydrolysis of the lactone ring of DHA. Time course of degradation of 3,4-End DKGL was followed by HPLC monitoring at $245 \mathrm{~nm}$. The measurement was carried out after $\mathrm{N}_{2}$

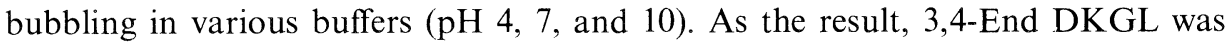
found to be quite stable at $\mathrm{pH} 4$. But, 3,4-End DKGL degraded about $50 \%$ after 4 -h bubbling at $\mathrm{pH} \mathrm{7,} \mathrm{whereas} \mathrm{it} \mathrm{degraded} \mathrm{almost} \mathrm{completely} \mathrm{in} \mathrm{2-h} \mathrm{bubbling} \mathrm{at} \mathrm{pH} 10$.

\section{Reduction of DHA by 3,4-End DKGL}

Another portion of the syrup of 3,4-End DKGL was diluted variously with phosphate buffer ( $\mathrm{pH}$ 7.2). The initial concentration of 3,4-End DKGL was determined by the optical density at $265 \mathrm{~nm}$ with the assumption that the absorbance of 3,4-End DKGL was identical with that of AsA (3,4). To a portion of this solution was added DHA aqueous solution to make a final concentration of 2.5 or $5 \mathrm{~mm}$, and this was incubated at $37^{\circ} \mathrm{C}$ for $1 \mathrm{~h}$. Table 2 shows the relationship between the concentrations of 3,4-End DKGL added and the formed AsA estimated by HPLC. Addition of $1.5 \mathrm{~mm} 3,4-$ End DKGL to the solution of DHA $(2.5 \mathrm{~mm})$ was found to form about $0.15 \mathrm{~mm}$ AsA: the formed AsA corresponds to only $10 \%$ of 3,4-End DKGL used in the presence of excess DHA concentrations; at a lower concentration of 3,4-End DKGL described above, the reduction ratio of DHA to AsA was somewhat higher (about 20\%). The conversion ratio was thus low, but the possibility of the formation of AsA from DHA without other reducing agent is interesting, if the system has no oxygen.

Reaction of 3,4-End DKGL with o-phenylenediamine (OPD)

To an acidic solution ( $\mathrm{pH} 4$ ) of 3,4-End DKGL was added a 4-fold excess of 
Table 2. Reduction of DHA to AsA by 3,4-End DKGL in phosphate buffer (pH 7.2) under $\mathrm{N}_{2}$ bubbling at $37^{\circ} \mathrm{C}$ for $1 \mathrm{~h}$.

\begin{tabular}{ccc}
\hline $\begin{array}{c}\text { DHA solution } \\
(\mathrm{mM})\end{array}$ & $\begin{array}{c}\text { 3,4-End DKGL added } \\
(\mathrm{mM})\end{array}$ & $\begin{array}{c}\text { AsA formed } \\
(\mathrm{mM})\end{array}$ \\
\hline 2.5 & 0 & - \\
& 0.15 & 0.027 \\
& 0.75 & 0.091 \\
5.0 & 1.50 & 0.149 \\
& 0 & - \\
\hline
\end{tabular}

The initial concentration of 3,4-End DKGL was determined by the optical density at $265 \mathrm{~nm}$. The concentration of AsA formed was measured by HPLC.

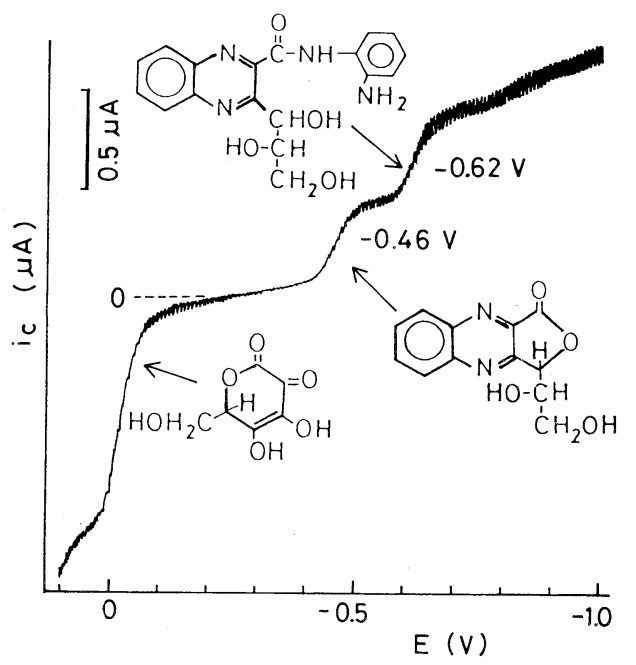

Fig. 4. Polarogram of condensation products of 3,4-End DKGL with OPD. 3,4-End DKGL was dissolved in acetate buffer $(\mathrm{pH} 4)$ under $\mathrm{N}_{2}$ bubbling, and 4-fold excess of OPD was added to the solution. After standing for $50 \mathrm{~h}$ at $37^{\circ} \mathrm{C}$ under $\mathrm{N}_{2}$ bubbling, this sample was taken for the polarographic limiting current $v$. S.C.E.

$\mathrm{OPD}$, then this was incubated at $37^{\circ} \mathrm{C}$ for $50 \mathrm{~h}$ under $\mathrm{N}_{2}$ bubbling. A portion of the reaction mixture was withdrawn, then analyzed by polarography (7). From the halfwave potential of the sample as is shown in Fig. 4, one quinoxaline having $E_{1 / 2}=$ $-0.62 \mathrm{~V} v s$. a saturated calomel electrode (S.C.E.) was found to be 2-(2aminophenylcarbamoyl)-(1' $\left.S, 2^{\prime} R\right)-3-\left(1^{\prime}, 2^{\prime}, 3^{\prime}\right.$-trihydroxypropyl)quinoxaline, which is condensed at the C-2 and C-3 of 3,4-End DKGL, and also split at the lactone ring, followed by an acid-amide reaction with OPD, as reported previously (12). The other one having $E_{1 / 2}=-0.46 \mathrm{~V}$ vs. S.C.E. was found to be $\left(1^{\prime} S\right)-2-\left(1^{\prime}, 2^{\prime}-\right.$ dihydroxyethyl-1-yl)quinoxaline-2-carboxylic acid $\gamma$-lactone, which is condensed at 


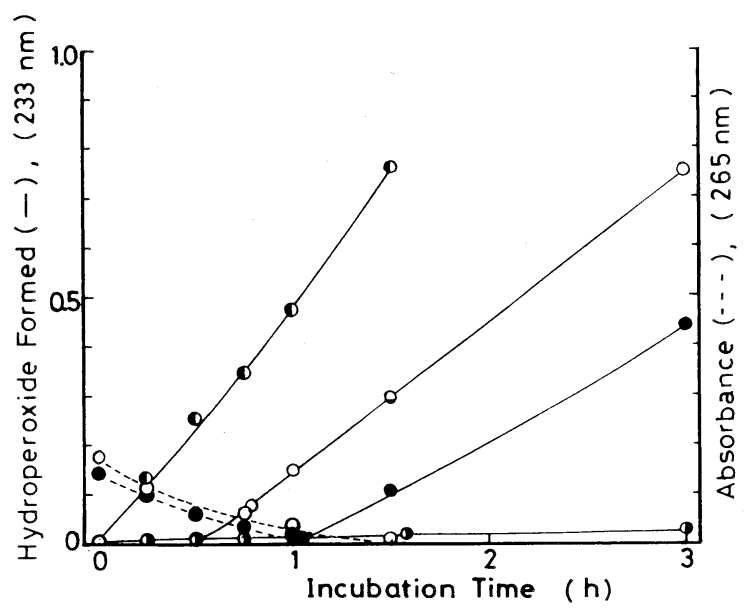

Fig. 5. Effects of 3,4-End DKGL on linoleic acid peroxidation containing $20 \% \mathrm{EtOH}$ and $10 \mathrm{~mm}$ LA in phosphate buffer $(\mathrm{pH} 7.0)$. A portion $(0.3 \mathrm{ml})$ of a reaction mixture $(5 \mathrm{ml})$ containing 3,4-End DKGL, AsA or DHA, $10 \mathrm{~mm} \mathrm{LA}$, and $20 \% \mathrm{EtOH}$ in phosphate buffer was withdrawn, and added to $3 \mathrm{ml}$ of $60 \%$ EtOH solution containing $200 \mathrm{ppm}$ EDTA, then UV spectra were measured. The solid lines show the absorbance at $233 \mathrm{~nm}$, and broken lines, absorbance at $265 \mathrm{~nm}$. D, DHA $(135 \mu \mathrm{M})$; $\bigcirc$, AsA $(135 \mu \mathrm{M}) ; \bullet$, 3,4-End DKGL $(110 \mu \mathrm{M})$; and $\mathbf{-}$, control without catalyst.

the C-2 and C-3 positions of DHA or 3,4-End DKGL. But, most of the 3,4-End DKGL added to the solution remained unreacted. This may suggest that one or two of the carbonyl residues of 3,4-End DKGL might be very firmly hydrated in the acidic solution used. Therefore, the condensation reaction of 3,4-End DKGL with OPD might not proceed, differing from the usual DHA analogs, as reported previously (12).

\section{Effects of 3,4-End DKGL on LA peroxidation}

3,4-End DKGL was added to test LA peroxidation in the reaction mixture containing $20 \% \mathrm{EtOH}$ and $10 \mathrm{~mm} \mathrm{LA}$. As is obvious from Fig. 5, in the presence of the catalyst, DHA accelerated the LA peroxidation with time from the start of the incubation. Addition of AsA $(135 \mu \mathrm{M})$ suppressed the LA peroxidation at least for $30 \mathrm{~min}$ after the incubation, and then accelerated it gradually, but addition of $110 \mu \mathrm{M} 3,4-E n d$ DKGL suppressed the LA peroxidation more clearly than did AsA. After the suppression of LA peroxidation had been ended, it changed to accelerate LA peroxidation gradually, and the rate of autoxidation of LA catalyzed by 3,4End DKGL was similar to that catalyzed by AsA. This may mean there is acceleration of $\mathrm{LA}$ peroxidation caused by the active oxygens after the consumption of 3,4-End DKGL. Therefore, 3,4-End DKGL and its degraded species were important either as scavengers or accelerators of LA peroxidation. 


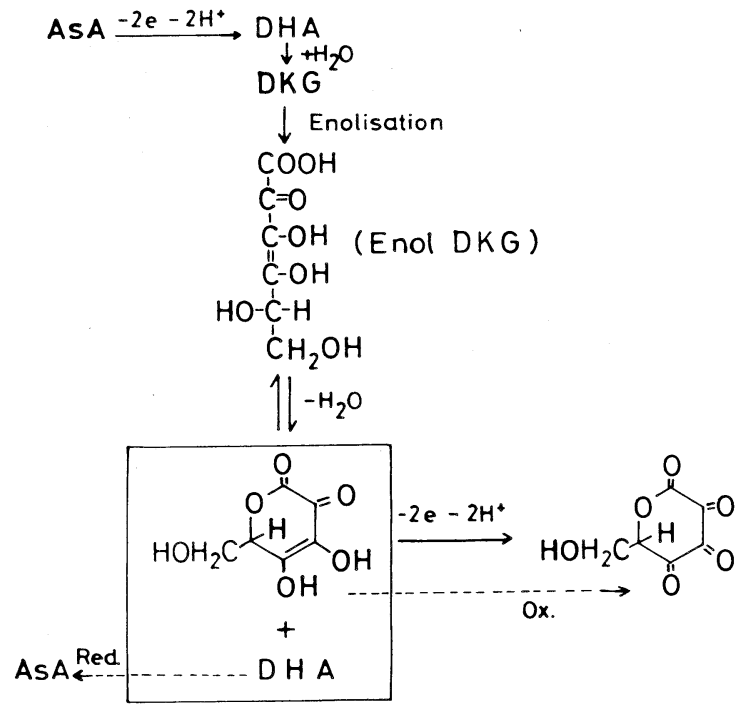

Fig. 6. Relations between electrode oxidation, spontaneous degradation of AsA, and reduction of DHA by 3,4-End DKGL in phosphate buffer ( $\mathrm{pH} 7.2$ ) under $\mathrm{N}_{2}$ bubbling. The vertical lines show the spontaneous degradation, and horizontal lines, electrode oxidation of the materials.

From these results above, we can conclude as follows. As is shown in Fig. 6, AsA is easily oxidized to DHA, followed by hydrolysis of the lactone ring to form DKG. About $10 \%$ of the DKG is lactonized to form 3,4-End DKGL in the neutral solution under $\mathrm{N}_{2}$ bubbling. The 3,4-End DKGL thus formed reduces DHA to AsA in neutral to weakly alkaline solutions. 3,4-End DKGL, which has three carbonyl groups, is unstable. The di- or tri-carbonyl group may be easily hydrated in neutral or weakly alkaline solutions, so the lactone ring splits hydrolytically via di- or tricarbonyl intermediates.

AsA is formed from mono-DHA by a disproportionation reaction. Also, acireductones such as triose-reductone and reductic acid are formed from AsA or sugars by pyrolysis $(13,14)$. However, AsA was formed from DHA or DKG with $\mathrm{N}_{2}$ bubbling under the present experimental conditions. The fact that 3,4-End DKGL reduced DHA to AsA in a physiologically common $\mathrm{pH}$ solution, may be interesting in relation to the action of DHA, which has been discussed to be effective as one-half of the action of vitamin $\mathrm{C}$ in our body fluids or blood. Hitherto, DHA has been reported to be reduced to AsA by glutathione in the blood $(15,16)$. In this paper we like to suggest a possibility of the above-stated reduction of DHA to AsA by 3,4-End DKGL which can be produced from DHA or DKG under $\mathrm{N}_{2}$ bubbling. In this connection, to clarify the behavior of 3,4-End DKGL further in vivo, methods for stable and easy preparation of the sample are urgently required, since 3,4-End DKGL has not been easy to prepare on a large scale. 


\section{REFERENCES}

1) Takagi, M., Higashioka, H., Tamura, K., and Morita, N. (1986): Effects of L-ascorbic acid and dehydro-L-ascorbic acid on the peroxidation of linoleic acid in neutral phosphate buffer containing alcohol. Agric. Biol. Chem., 50, 41-47.

2) Takagi, M., Onodera, H., Miyamoto, I., and Morita, N. (1988): Effects of active oxygen scavengers on the peroxidation of linoleic acid catalyzed by dehydro-L-ascorbic acid or its degradation products. J. Nutr. Sci. Vitaminol., 34, 141-149.

3) Otsuka, M., Kurata, T., and Arakawa, N. (1986): Isolation and characterization of a degradation product derived from 2,3-diketo-L-gulonic acid, in Amino-Carbonyl Reactions in Food and Biological Systems, ed. by Fujimaki, M., Namiki, M., and Kato, H., Developments in Food Science, Vol. 13, Elsevier, Amsterdam, pp. 77-84.

4) Otsuka, M., Kurata, T., and Arakawa, N. (1986): Isolation and characterization of an intermediate product in the degradation of 2,3-diketo-L-gulonic acid. Agric. Biol. Chem., 50, 531-533.

5) Sawer, D. T., Chiericato, G., Jr., and Tsuchiya, T. (1982): Oxidation of ascorbic acid and dehydroascorbic acid by superoxide ion in aprotic media. J. Am. Chem. Soc., 104, 6273-6278.

6) Kagawa, Y. (1962): Enzymatic studies on ascorbic acid catabolism in animals. 1. Catabolism of 2,3-diketo-L-gulonic acid. J. Biochem., 51, 134-144.

7) Takagi, M., and Morita, N. (1987): Active oxygens and the peroxidation of linoleic acid catalysed by degraded species of ascorbic acid. Bioelectrochem. Bioenerg., 18, 171-178.

8) Takagi, M., Morita, N., Kawajiri, A., Sasai, K., and Sawada, S. (1988): Electrochemical properties of intermediate products of dehydroascorbic acid and 2,3diketo-L-gulonic acid. Rev. Polarogr., 33, 65.

9) Takagi, M., Kawajiri, A., Morita, N., Sawada, S., and Mizunami, Y. (1987): Electrochemical properties of degraded products from dehydroascorbic acid and in neutral and weakly acidic solutions, in Abstracts of papers, The Annual Meeting of Agricultural Chemical Society of Japan, p. 784.

10) Tolbert, B. M., and Ward, J. B. (1982): Dehydroascorbic acid, in Ascorbic Acid: Chemistry, Metabolism, and Uses, ed. by Seib, P. A., and Tolber, B. M., Advances in Chemistry Series 200, American Chemical Society, Washington, D. C., pp. 101-123.

11) Cooke, J. R., and Moxon, R. E. D. (1981): The detection and measurement of vitamin C, in Vitamin C (Ascorbic Acid), ed. by Counsell, J. N., and Hornig, D. H., Applied Science Publishers, London, pp. 167-198.

12) Tsujimoto, Y., Ohmori, M., and Takagi, M. (1985): Condensation of $o$ phenylenediamine with dehydro-L-ascorbic acid derivative and analogs. Carbohydr. Res., 138, 148-152.

13) Matsui, G., Obata, H., Shikata, K., and Ueno, T. (1978): Formation of reductones from cellulose by pyrolysis. Nippon Nögeikagaku Kaishi (Agric. Chem. Soc. Jpn.), 52, 69-73.

14) Weis, W., Zubrzycki, Z., and Staudinger, H. (1968): Redox behavior of L(+)semidehydroascorbic acid. Hoppe-Seyler's Z. Physiol. Chem., 359, 279-282.

15) Stankova, L., Rigas, D. A., and Bigley, R. H. (1975): Dehydroascorbate uptake and reduction by human blood. Nucl. Sci., 10, 31 .

16) Bates, C. J. (1981): The function and metabolism of vitamin $C$ in man, in Vitamin C (Ascorbic Acid), ed. by Counsell, J. N., and Hornig, D. H., Applied Science Publishers, London, pp. 1-22. 